\title{
Les attitudes d'apprenants taiwanais de langue étrangère à l'égard de la bande dessinée et quelques implications
}

\author{
Laurent-Fidèle Sossouvi (Kaohsiung, Taiwan)
}

\begin{abstract}
The issue of exploitation foreign languages' instructional comics has been widely discussed in recent years. However, although the authors indicate its advantages and its great educational potential, few have actually investigated learners' attitudes towards this learning resource. And everyone knows the importance of affective factors in the process of learning foreign languages. In addition, comic is rarely used as a teaching aid in Asia. As part of this contribution exploratory, we propose to examine not only the attitudes of Sinophones Learners of French as Foreign Language towards comic, but also the support it can provide. To achieve this goal, fifty-five Taiwanese learners, from level A1 to B1, divided into three distinct groups were probed, questioned and subjected to work around comics. According to the results, we conclude that (i) learners have positive attitudes towards comics, mangas, and are highly motivated; (ii) the use of comics has helped to improve their language skills and intercultural learning as well as revitalize the target language. Implications of these findings are discussed.
\end{abstract}

\section{$1 \quad$ Introduction}

Cette contribution a pour objet principal de mettre en lumière les attitudes des apprenants universitaires de FLE à l'égard de la bande dessinée (BD), afin d'énumérer quelques possibilités d'exploitation pédagogique. Il existe une littérature abondante montrant que son utilisation comme outil pédagogique formel offre plusieurs atouts pouvant promouvoir les processus d'enseignement/apprentissage, voire contribuer au développement durable de compétence (voir Barron-Carvais 1994 ; Morlat/Tomimoto 2007 ; Djamel 2007, par exemple). Pourtant, l'insertion de ce document authentique dans l'enseignement des langues étrangères reste encore modeste, surtout dans l'enseignement du FLE à Taiwan. De même, peu de recherches se sont intéressées aux attitudes d'apprenants à son égard. On privilégie beaucoup plus les Technologies de l'information et de la communication (TIC), l'Internet et les ressources d'Internet. C'est ainsi que dans la littérature, les études sur les attitudes des apprenants à l'égard d'Internet foisonnent (par exemple Kasanga 1996 ; Slate et al. 2002 ; Usum 2003 ; Yang/Chen 2007, entre autres). Or, l'utilisation de la BD, ce moyen d'expression universelle, pourrait également ouvrir des horizons didactiques plus larges, permettant à l'apprenant d'acquérir plus d'autonomie, plus d'assurance dans son apprentissage, d'apprendre de façon divertissante et surtout le développement de ses capacités linguistiques, interculturelles, socio-pragmatiques.

Mentionnons que cet article n'est qu'une ébauche à compléter. Dans les lignes qui suivent, il sera proposé sommairement, de prime abord, l'ancrage théorique qui sous-tend cette étude. Ensuite, il sera présenté la méthode de recherche appliquée, où nous tenterons de parler des informateurs, des matériaux et de la procédure utilisée. Enfin, nous envisagerons les principaux résultats, leurs interprétations et quelques pistes pédagogiques. 


\section{Quelques considérations préliminaires}

Avant d'entrer dans le vif du sujet, essayons d'explorer les grandes lignes de l'environnement dans lequel se déroule l'apprentissage de la langue étrangère (LE), justifier notre démarche, pour terminer par ce que recouvre le concept d'attitude, qui apparaît de plus en plus dans la littérature.

Taiwan a pour langue officiel unique le chinois mandarin, langue dominante sur le plan éducatif, politique, culturel étant donné qu'elle est la langue de l'enseignement, de la communication publique, des médias, etc. Les langues européennes comme l'anglais, le français, l'espagnol et l'allemand font aussi partie intégrante des programmes scolaires du pays. Le chinois mandarin et les langues étrangères de grande diffusion antérieurement mentionnées ne sont pas apparentés. En effet, ces langues appartiennent à différentes familles linguistiques : le chinois mandarin est classée dans la famille sino-thaïe, alors que l'anglais, l'allemand sont des langues germaniques, et le français, l'espagnol, des langues romanes. De même, le premier utilise des sinogrammes alors que les langues européennes ont recours à l'alphabet latin. Il est aussi à noter que les autres langues locales comme le tai-yu et le hakka appartiennent à la même famille que le chinois mandarin ; les langues aborigènes sont des langues austronésiennes. Un rapide tour d'horizon montre la situation fragile des langues locales pas assez apprises dans le système scolaire. En revanche, la langue anglaise est présente partout à Taiwan et occupe, de nos jours, une position dominante dans le système éducatif taïwanais (cf. Executive Yuan 2003), voire dans la société taïwanaise. Huang (2005: 48) résume ainsi la situation de la langue de Shakespeare dans l'île :

English, a foreign language to the Taiwanese linguistic majority, has become an integral part of Taiwan's economic, financial, and technological development. It is also a leading factor in educational success of students in Taiwan who are learning English from an increasingly younger age.

Autrement dit, l'anglais peut être considéré dans le pays comme une sorte de langue seconde (L2), du moins, une langue moins étrangère que les autres langues non maternelles enseignées, dans la mesure où elle demeure la langue dominante des échanges internationaux autant sur la scène économique que diplomatique. Pour aller plus loin, on peut même la considérer comme une seconde langue officielle dans l'île. Cette pression de l'anglais dans le système éducatif menace l'apprentissage des autres langues étrangères. Toutefois, le japonais occupe également une place non négligeable pour des raisons tant historiques, économiques que géographiques. Certaines langues européennes telles que le français, l'allemand, l'espagnol sont aussi en pleine ascension dans de nombreux centres d'enseignement. Les deux premières langues sont les langues européennes les plus populaires, les plus étudiées (Lin 2007 : 29). Tout compte fait, à Taiwan, plusieurs langues de statuts différents cohabitent. ${ }^{1}$

Signalons également que mis à part la distance linguistique, il existe une importante distance géographique, voire culturelle entre l'île de Taiwan et les pays de la communauté des locuteurs natifs, par exemple des langues romanes, telles que le français et l'espagnol. Effectivement, aucun voisin immédiat de l'île n'a le français ou l'espagnol comme langue officielle ; par conséquent, les apprenants l'apprennent dans le cadre restreint de la salle de classe, d'où de nombreuses ruptures entre la classe de LE et la dynamique interactive de la vie quotidienne. Le développement de leur compétence sociolinguistique et pragmatique se voit donc très limité (Ellis 1992 ; Tarone 2000). Et on peut observer une plus forte proportion d'erreurs pragmatiques (cf. Romero Trillo 2002). Ils peuvent ainsi demeurer " monostylistiques " dans la langue cible (Gass et al. 1998 ; Dewaele/Regan 2002). Ainsi, la seule compétence linguistique ne suffit plus. De plus, l'apprenant contemporain, notamment taïwanais, est un enfant de la civilisation de l'image, caractérisé par une attention fragile et un manque de concentration.

\footnotetext{
${ }^{1}$ Pour plus de détails sur la situation linguistique du pays, voir Saillard (1998), Lin (2007), Sossouvi (2010).
} 
Par ailleurs, malgré la signification extraordinaire acquise depuis quelques années par les TIC en milieu scolaire, la documentation scientifique récente suggère qu'il y a encore une très faible proportion d'enseignants qui les intègrent réellement dans leurs pratiques pédagogiques (Rogers 2000 ; Schmid/Schimmack 2010). Ce recours limité semble être causé, du moins en partie, par leur illettrisme technologique, leur manque de compétences informatiques, la difficulté à réapprendre à apprendre à intégrer les TIC dans une perspective pédagogique, la nonperception de leurs usages par certains d'entre eux. Lewis (2010:9) abonde dans le même sens, soulignant également que " New technologies open up possibilities unheard of in previous. But these technologies will have only limited impact if the pedagogy behind their application does not keep pace ". De l'avis de Marton (1999), de plus en plus d'enseignants les utilisent beaucoup plus par la fascination de la nouveauté que pour des visées ponctuelles de formation; et il prévient de la tendance à considérer ces technologies comme une panacée à tous les maux dont souffre l'enseignement (des langues étrangères). Qui plus est, plusieurs chercheurs tels que Clack (1994), Wisher/Priest (1998), Russell (1999) soutiennent qu'il n'existe pas de différence significative entre un enseignement intégrant les TIC et un enseignement classique sans elles.

De même, comme le constatent plusieurs auteurs comme Leather (2003), Luis Banegas (2011), la majorité des manuels scolaires destinés à l'enseignement des langues étrangères manquent de réalisme ; ils les trouvent insipides, ennuyeux, peu attrayants et parfois mensongers.

À la lumière de ce qui précède, l'on peut estimer qu'une diversification des supports, des dispositifs d'enseignement, donc diversification de la pédagogie pourrait remédier un peu à la situation. Les modes d'apprentissage des langues sont innombrables et on peut s'appuyer sur des supports variés : textes, iconographies, images fixe et animée, multimédia. D'où notre idée d'examiner les attitudes d'étudiants universitaires sinophones apprenant le FLE à l'égard de la $\mathrm{BD}$, cette suite de dessins racontant une histoire. Nous pensons que cette dernière, avec ses nombreuses possibilités, peut permettre un apprentissage efficace de la langue française, le développement des compétences idiomatico-culturelles, tout en favorisant l'autonomie des apprenants. Mais avant d'en arriver là, essayons de mieux comprendre ce qu'on entend lorsqu'on parle d'attitude.

Au cours des dernières décennies, de nombreuses recherches ont été effectuées dans le but d'étudier les facteurs qui influencent positivement ou négativement l'apprentissage des langues. Il existe trois catégories de facteurs : les facteurs cognitifs, affectifs et situationnels. C'est principalement les avant-derniers qui nous intéressent dans cette étude, notamment les attitudes et émotions associées aux questions de la BD. Malgré une littérature abondante sur les attitudes, force est de constater que la plupart des définitions données par les chercheurs (par exemple Fazio 1986 ; Eagly/Chaiken 1993 ; Thomas/Alaphilippe 1993 ; Legendre 1993 : 112) sont complémentaires. C'est un concept-clé pour rendre compte de l'état d'esprit, des comportements d'un public. Si tout commence par l'éducation, tout s'esquisse également avec une attitude. En fait, le concept d'attitude réfère habituellement à l'ensemble des évaluations, des réponses émotionnelles par rapport à une communauté, des faits sociaux, des stimuli sociaux ou physiques, une personne, un objet, des habitudes ou des valeurs, etc. En résumé, c'est l'ensemble des dispositions mentales, des perceptions, des appréciations, des jugements qu'une personne porte sur tout élément de son environnement. Pour ce qui nous concerne, on peut donc considérer l'attitude comme un état d'esprit ou une disposition qui pousserait (ou non) à lire ou à aimer la bande dessinée. La majorité des chercheurs ont une vision unidimensionnelle de l'attitude (par exemple Fishbein/Ajzen 1975 ; Ajzen/Fishbein 1977, 1980 ; Petrof 1999). Pour ces auteurs, un individu évalue un objet soit positivement, soit négativement. De façon générale, les évaluations peuvent être positives (des comportements d'approche), négatives (des comportements d'évitement, de rejet) ou neutres (la personne ne fait aucune associa- 
tion positive ou négative avec la réalité qui l'entoure). Dans le domaine des langues et de l'éducation multilingue, les attitudes constituent l'une des composantes majeures de la motivation dans les taxonomies de certains chercheurs comme Gardner (1985), Clément et al. (1994), Dörnyei (1994). Et bien qu'elles soient difficilement mesurables d'une façon rationnelle et méthodique, elles ont toujours été ressenties comme une condition importante dans le processus d'apprentissage des langues. De nombreux auteurs (par exemple Potvin/Paradis 2000) ont démontré que les attitudes des apprenants envers un objet, un individu, un phénomène social, etc., déterminent leurs comportements et leurs performances aussi bien affectives que cognitives. Il en résulte, par exemple, que les attitudes positives s'accompagnent souvent de bons résultats linguistiques, d'autonomie, d'une forte motivation et de persévérance dans l'apprentissage de la langue cible.

\section{$3 \quad$ Méthode}

Dans la section précédente, nous avons brossé sommairement le contexte d'appropriation qui a été celui des participants étudiés et défini le concept d'attitude. Dans la présente section, nous tenterons de présenter la méthodologie adoptée dans cette recherche empirique. Nous commencerons par une brève présentation des caractéristiques de la population étudiée, la collecte de donnée, pour terminer par le déroulement de la recherche.

\subsection{Les participants}

Cinquante-cinq étudiants universitaires $(n=55)$, dont environ 45 étaient des filles, prirent part à l'étude. Les participants sont repartis en trois groupes, de niveau A1 à B1, d'une université située à Kaohsiung, la deuxième plus grande ville de Taiwan. Ils étaient âgés de 19 à 22 ans . Le groupe G1 était composé de 20 apprenants taïwanais (dont 15 filles et 5 garçons) de 19 à 20 ans. Ils étaient en première année d'apprentissage de la langue française. Le groupe G2 se composait de 20 apprenants (16 filles et 4 garçons) de deuxième année de FLE, âgés de 20 à 21 ans. Le groupe G3 comprenait 15 apprenants (14 filles et un garçon), dont l'âge varie entre 20 à 22 ans, bénéficiant de 5 ans d'étude de la langue de Molière. Tous les participants de l'étude ont le même arrière-plan culturel et linguistique, ils étaient de langue maternelle chinoise (certains d'entre eux parlent en plus le taiyu et/ou le hakka). Ils avaient appris la langue anglaise pendant 8 à 13 ans, en conséquence, ils avaient atteint le niveau de compétence avancé ou supérieur. Certains d'entre eux se sont rendus dans des pays anglophones et francophones en vue d'apprendre la langue ou pour faire du tourisme. Mentionnons en outre qu'ils ont commencé l'apprentissage du FLE en tant que vrais débutants, et qu'ils recevaient neuf heures d'enseignement de cette langue par semaine. Par ailleurs, ils ont rarement l'occasion de s'engager dans des interactions authentiques avec les locuteurs natifs de la langue cible en dehors de la salle de classe. Le tableau 1 présente la répartition des sujets de l'échantillon par sexe et par niveau selon le groupe d'appartenance.

\begin{tabular}{|l|l|l|}
\hline Catégorie & Taille de l'échantillon & Pourcentage $(\%)$ \\
\hline Groupe G1 & $20(15$ filles/5 garçons) & $36,36 \%$ \\
\hline Groupe G2 & $20(16$ filles/4 garçons) & $36,36 \%$ \\
\hline Groupe G3 & $15(14$ filles/1 garçon) & $27,28 \%$ \\
\hline Total & $55(45$ filles/10 garçons) & $100 \%$ \\
\hline
\end{tabular}

Tableau 1 : Répartition de l'échantillon

\subsection{Le matériel utilisé}

Les instruments utilisés dans le cadre de cette étude sont : (i) un questionnaire, (ii) des entretiens, (iii) un enregistreur numérique, (iv) des activités autour de la bande dessinée, (v) l'ob- 
servation, (vi) des planches de bandes dessinées, (vii) un carnet de notes dans lequel nous inscrivons les commentaires, les remarques éventuelles.

Le questionnaire comportait une quinzaine de questions sur leur biographie, la profession de leurs parents, leurs habitudes langagières et les attitudes à l'égard de la bande dessinée. Ces dernières étaient mesurées par quatre items, notamment : aimez-vous lire les bandes dessinées ? Pourquoi ? La lecture de BD vous aide-t-elle dans l'apprentissage des langues ? Pourquoi ?

Une recension des écrits sur le sujet permet d'affirmer que bon nombre de recherches ont utilisé également des questionnaires pour recueillir leurs données (par exemple Gardner 1985 ; Clément et al. 1994 ; Yager 1998 ; Tragant/Muñoz 2000 ; Sossouvi 2006, entre autres).

Les entretiens portaient également sur des questions ayant trait à la bande dessinée : par exemple, lisez-vous des bandes dessinées ? Pourquoi ? Qu'aimez-vous dans les bandes dessinées? Ils nous ont permis un contact direct avec les participants, nous apportant la certitude que leurs réponses étaient individuelles, et qu'ils ne sont pas influencés en communicant entre eux.

Les tests et les activités ont été administrés en fonction du niveau du groupe afin d'évaluer l'efficacité de la BD comme support à l'apprentissage de langues étrangères. Les planches de BD ont été tirées aussi bien d'ouvrages scolaires (Salins/Dupré La Tour 1988 ; Lescure 2006, etc.) que d'albums (Astrix, de Tintin, de Spirou et Fantasio, de T'choupi, de Titeuf). Chaque groupe a réalisé au moins huit tâches : deux activités sur les textes de BD sans les images, mais avec des questions de compréhension; deux activités à partir de la BD complète (texte et image) suivie de questions de compréhension (pour saisir le sens général du document et des questions de compréhension fine), deux activités de lecture de compréhension de bandes dessinées, deux exercices pour remplir et imaginer les dialogues des personnages de BD ainsi que deux activités pour choisir le dialogue correspondant à chaque vignette. Mentionnons que chaque activité comportait au moins une dizaine d'items et que le mode de réalisation était individuel.

\subsection{La procédure}

Le recueil de données s'est fait dans un intervalle d'une année académique, soit de septembre 2009 à juin 2010. Le questionnaire a été rempli en début d'année universitaire, durant les heures de cours, en un temps moyen de 20 à 25 minutes, les plus rapides en 16 à 17 minutes, les plus lents en 25 et 30 minutes. Nous leur avions signalé que c'était une enquête de routine pour mieux les connaître. Les entretiens semi directifs auprès d'une vingtaine d'entre eux constituent une importante source d'information, ils duraient généralement entre cinq et dix minutes, et nous ont permis d'isoler leurs attitudes à l'égard de la BD. Certains entretiens ont été enregistrés à leur insu et transcrits avant d'être analysés. Durant cette période, toutes les activités ont été réalisées dans la salle de classe pendant les heures de cours, sur une durée allant de 30 à 45 minutes. Les apprenants ont complété des questionnaires à choix multiples, sur la compréhension globale. Ils ont été amenés à associer des dialogues à des vignettes, compléter des dialogues à l'aide d'informations fournies, voire identifier les intentions et les sentiments des personnages, les registres de langue. Voici quelques consignes données pour faire les exercices : À partir de la planche de BD suivante, retrouvez l'histoire en remettant les vignettes dans l'ordre. Imaginez les paroles de chaque vignette. Faites la description des personnages de cette BD ... Imaginez la situation, etc. Après avoir donné les consignes, nous demandons à certains participants de les reformuler avec leurs propres mots, afin de voir s'ils ont tout compris de la même manière. Les différentes activités ont été corrigées par nos soins. Le lecteur trouvera en annexe quelques exemples de copies de nos sujets. 


\section{$4 \quad$ Présentation et interprétation des résultats}

Nous nous bornerons ici à rendre compte d'une partie des résultats, ceux ayant trait à leurs attitudes globales à l'égard de la bande dessinée et ceux concernant les activités. Enfin, nous allons tenter d'interpréter les résultats au regard des questions soulevées par la présente contribution ainsi que proposer quelques pistes pédagogiques.

\section{1 À propos des différents types d'attitudes}

\begin{tabular}{|l|l|l|l|l|}
\hline Attitudes & Groupe G1 & Groupe G2 & Groupe G3 & Total \\
\hline Positives & 18 & 17 & 10 & $45(81,81 \%)$ \\
\hline Négatives & 01 & 02 & 01 & $04(07,27 \%)$ \\
\hline Ambivalentes & 00 & 00 & 04 & $04(07,27 \%)$ \\
\hline Neutres & 01 & 01 & 00 & $02(03,63 \%)$ \\
\hline Total & 20 & 20 & 15 & $55(100 \%)$ \\
\hline
\end{tabular}

Tableau 2 : Récapitulatif des attitudes identifiées

Sans être exhaustif, le tableau ci-dessus présente les différentes attitudes manifestées par nos participants. Les résultats permettent de distinguer quatre types d'attitudes.

\subsubsection{Des attitudes positives}

Comme on peut le constater sur le tableau 1, les attitudes de nos participants à l'égard de cet outil d'aide à l'apprentissage sont, dans l'ensemble, positives. En effet, 18 des sujets sur $20 \mathrm{du}$ groupe G1, 17 sur 20 des participants du groupe G2 et 10 sur 15 des sujets du groupe G3 ; autrement dit $81,81 \%$ de notre échantillon aiment et apprécient les bandes dessinées : " lire des bandes dessinées, c'est formidable ". Dans les raisons évoquées par nos participants " j'ai amélioré mon anglais grâce aux BD ", " c'est grâce aux mangas que je parle mieux japonais ", " je lis les BD parce que cela me plaît ", " j'adore les mangas, elles me rendent très heureuse " reviennent le plus souvent. Ces attitudes favorables sont donc attribuables au plaisir ${ }^{2}$ qu'ils tirent de la lecture de la BD, l'humour qu'elle véhicule, en d'autres termes, l'aspect distrayant et ludique, voire son rôle facilitateur et stimulant pour l'apprentissage des langues. Une autre explication plausible serait notamment la résultante de l'association entre les bandes dessinées et les stimuli positifs, comme des expériences heureuses, l'identification aux héros de $\mathrm{BD}^{3}$, le jeu simultané de dessins et textes, la lecture de cet outil depuis l'enfance, etc. Autrement dit leurs attitudes agréables résultent de la nature hédonique, utilitaire de la BD et leur contentement lors des différentes lectures. Cela signifie que les jeunes apprenants manifestent encore d'enthousiasme pour ce neuvième art ; ce qui confirme les affirmations de Darao (2004 : 596) selon lesquelles la BD implique toutes les générations et qu'elle est acceptée par tout le monde. De la même façon, ce résultat met en exergue la fonction instrumentale, utilitaire ou pragmatique des attitudes. Il ressort aussi que les apprenants intéressés par un outil, un objet ou une activité d'apprentissage manifestent des affects et une évaluation favorable à son endroit. Ce résultat peut être rapproché d'une étude de Galvis (2010) qui a détecté des attitudes favorables envers le bilinguisme anglais-espagnol chez 87,5\% des participants. Par ailleurs, comme l'indiquent certains chercheurs (par exemple Larsen-Freeman/Long 1994 ; MarcosLlinas 2007), il y a des corrélations significatives entre les attitudes positives et une motivation plus élevée, les résultats linguistiques et la persévérance dans l'apprentissage de la langue cible. Cette attitude positive envers la BD reflète une réelle motivation, l'enthousiasme et une prédisposition favorable à cet outil.

\footnotetext{
${ }^{2}$ Et ce plaisir est essentiel dans l'acquisition de langue étrangère, voire du goût de lire.

${ }^{3}$ S'identifier à des héros familiers peut favoriser l'apprentissage de langue étrangère, notamment la lecture.
} 


\subsubsection{Des attitudes négatives}

Toutefois, quatre participants (un sujet du groupe G1, deux sujets du groupe G2 et un sujet du groupe G3, soit $07,27 \%$ de notre échantillon ont manifesté une attitude globale négative ou défavorable à l'égard de la BD : " il y a trop de violence, trop de cruauté ", " Je déteste lire les $\mathrm{BD}$, je préfère le cinéma ", " c'est pour les enfants ", la cherté des BD. Cette désaffection à l'égard des BD s'expliquerait par leur âge, le déplaisir, le manque de ressources matérielles, de temps, la peur de la dépense inutile, la mentalité rétrograde de certains parents encore hostiles à toute idée de lire une $\mathrm{BD}$ (celle-ci étant vue comme une lecture frivole et triviale ${ }^{4}$ ), l'incidence de la panoplie d'activités culturelles, sociales et sportives. En effet, la plupart du temps, l'enfant possède des repères identitaires familiaux, et il adopte un comportement s'il sent que son entourage lui accorde de la valeur. En plus, l'apprenant d'aujourd'hui dispose d'un large éventail de loisirs sportifs, culturel, artistique, etc., à la fois attrayant et accessible, qui concurrence sérieusement la lecture des bandes dessinées. L'existence permanente de télévision, bluray, d'Internet, les réseaux sociaux, bref la prolifération des moyens de communication dans l'environnement de l'apprenant contemporain contribue certainement à ce qu'il lise moins ou déteste ce genre textuel. En plus, selon certaines études (Osborne/Collins 2000 ; Campbell 2001, par exemple), les activités de lecture sont mal perçues par les apprenants, elles sont vues comme des tâches totalement futiles et rébarbatives. De la même façon, le besoin de se différencier de ses compagnons, d'être unique expliquerait aussi ce rejet. En fait, lorsque l'apprenant se sent comparable aux autres condisciples, il cherche parfois à se distinguer d'eux, une sorte de protestation contre la similitude, dans le but de restaurer l'estime de soi. Une autre explication plausible est que, ces sujets jugent inutile ou inintéressante la BD. Ces résultats corroborent les constats faits par Cloutier (2003), Sossouvi (2006), Galvis (2010) qui rapportent également des attitudes négatives de la part de leurs sujets. De même, ils se rapprochent des études de Havard (1996), Sjoberg (2000), Postel-Vinay (2002), Venturini (2004) qui ont relevé chez plusieurs de leurs participants des attitudes négatives à l'égard des sciences.

\subsubsection{Des attitudes ambivalentes}

Les attitudes ambivalentes sont spécifiques à quatre participants du groupe G3 : " j'aime et je n'aime pas les BD ", " oui et non ". La désaffection envers la BD est corrélée avec de fausses croyances (violence, lecture frivole, " c'est pour les enfants ") alors que l'attitude favorable est corrélée avec les bénéfices utilitaires et hédoniques (l'aspect amusant et esthétique des BD). Ce résultat suggère qu'on peut trouver chez le même individu la coexistence de deux types d'attitudes opposées, des attitudes entremêlées. Cette accessibilité simultanée des aspects positifs et négatifs de la $\mathrm{BD}$ dénote une certaine maturité ; ils affrontent la réalité de manière dialectique, avec un esprit critique. On peut supposer qu'ils ont une préférence pour la cohérence, une tendance au besoin de cognition (Cacioppo/Petty 1982) et à la peur de l'approximation. Il est fort probable qu'une influence de leur trait de personnalité, de leur éducation, de leur expérience est à l'origine de cette ambivalence attitudinale. Ces données coïncident avec celles obtenues par Jonas et al. (1997), Conner/Sparks (2002), Armitage (2003) qui ont relevé chez leurs sujets des évaluations à la fois positives et négatives envers un unique produit. Toutefois, ces résultats vont à l'encontre des tendances de certaines théories des attitudes (par exemple Fazio 1989 ; Eagly/Chaiken 1993, Petrol 1999) en ce qui concerne la structure unidimensionnelle des attitudes, c'est-à-dire allant du négatif au positif. Cette vision bicéphale des attitudes ne reflète que partiellement les réalités attitudinales dans la vie : l'existence d'accessibilité simultanée des évaluations positives et négatives envers le même objet. On peut tout de même se demander si ce sentiment d'ambivalence ne cache pas aussi un type de neutralité, de modération ou d'indifférence.

\footnotetext{
${ }^{4}$ Alors qu'il existe de nos jours, de très bons albums qui conjuguent qualité littéraire et bonheur des yeux.
} 


\subsubsection{Des attitudes neutres}

Deux apprenants des groupes G1 et G2 ont affiché des attitudes neutres : " Je ne sais pas, je ne sais quoi dire ", " pas d'opinion ". Celles-ci pourraient être une conséquence de l'influence du contexte culturel, plus précisément des préceptes du confucianisme et du taoïsme qui les incitent à la souplesse, au non-dualisme, " ne rien faire ". En réalité, les traditions culturelles, les habitudes familiales peuvent influer sur les comportements, les attitudes. En conséquence, ils ont privilégié des attitudes objectives, dépassionnées. Peut-être tout simplement parce qu'ils considèrent qu'il ne faut pas manifester leurs émotions, surtout pas dans un centre de formation. Par ailleurs, il se pourrait que cette neutralité résulte du désintéressement, de l'incertitude, les apprenants n'arrivant pas à identifier leurs sentiments, à se décider. Ce résultat n'appuie pas l'idée de Petrof $(1999: 192)$ selon laquelle " Il ne peut y avoir d'attitude neutre ".

\section{2 À propos des activités}

S'agissant des activités autour des BD, les exercices sur le texte et sur la BD complète ont donné les résultats suivants. Les sujets du groupe G1 ont commis un total de 200 incorrections, soit 10 fautes par apprenant. Alors que pour l'activité sur la BD complète (image et texte), nous n'avons enregistré que 46 incorrections, soit 2,3 fautes par personne.

Pour ce qui a trait au premier type d'activité, les participants du groupe G2 ont fait 152 fautes, soit environ 7,6 fautes par personne. En revanche, 15 incorrections, soit 0,75 cas par apprenant, sont relevées dans l'exercice sur la BD complète. En somme, ils ont commis moins d'incorrections avec l'association images, dessins/écrit verbal.

Une interprétation possible pour ces bons résultats est que cette combinaison a produit un nouveau sens que ne produit pas l'écrit ou le dessin seul. En effet, la mise en forme bédéistique, ses caractéristiques expressives, etc., facilitent et éliminent la grande complexité des textes. Les sujets du groupe G3 n'ont pas réalisé ces tâches parce qu'ils les trouvaient assez faciles.

Quant aux deux activités qui demandaient de correspondre les bulles possibles aux images, nous avons enregistré dans le groupe G1, dix cas d'incorrections pour la première activité, et cinq réponses incorrectes au cours de la deuxième activité. Le groupe G2 a fait huit fautes et six fautes pour la deuxième activité. Enfin, le groupe G 3 a commis sept fautes au cours de la première activité et deux cas d'incorrections durant la passation de la deuxième activité (voir l'annexe 3, la copie du sujet Ch.).

En ce qui a trait aux deux activités qui consistaient à imaginer les paroles des vignettes en s'appuyant sur les images et les dessins, nous avons remarqué que les participants des trois groupes ont fait preuve d'imagination (voir par exemple les annexes 2 et 3), il y a peu d'énoncés problématiques. Les histoires reconstituées étaient logiques, adaptées aux contextes, et seuls deux apprenants du groupe G1 ont fait des contresens (voir annexe 1, la copie du sujet N.) : le sujet N. a mis " elle est aimable voir personnes de prison " au lieu de "Elle est aimable comme une porte de prison " ; " On dirait des completes " au lieu de " On dirait des sardines " ; " Mais quelle triste ! " au lieu de " Mais quelle idée ! ".

En ce qui concerne les deux activités de compréhension écrite à partir de bandes dessinées, les résultats indiquent que les participants des groupes G2 et G3 ont bien saisi le contenu, ils ont bien capté la trame générale, les subtilités de l'intrigue. Ces activités ont suscité également des débats très enrichissants qui nous ont permis de faire ressortir certains éléments de civilisation, de revoir, d'approfondir et d'employer les temps du passé. Par exemple, les critiques portaient sur la beauté des images-vignettes, les morales des histoires, la crédibilité ou le manque 
de crédibilité des faits rapportés, voire la discussion de différentes fins possibles aux scénarios. En effet, les images donnent souvent de nombreuses informations et détails.

Par comparaison à leur performance, pratiquement tous les apprenants ont obtenu des résultats relativement meilleurs. Il n'existe pas un lien significatif entre les attitudes des apprenants à l'égard de la BD et leurs performances. Toutefois, les participants qui ont affiché des attitudes positives ont obtenu de très bons résultats lors des diverses activités, ils se sont montrés disposer à faire des efforts, à persévérer et ont obtenu des scores plus élevés. Ensuite, la performance a tendance à baisser légèrement chez les sujets qui ont manifesté des attitudes ambivalentes, ceux qui ont présenté des attitudes neutres, et enfin, chez ceux qui ont affiché des attitudes négatives.

Ces résultats, globalement satisfaisants, soulignent combien il est intéressant d'intégrer le plus possible de matériels authentiques dans l'enseignement des langues étrangères. Ils suggèrent également que l'image est complémentaire du texte et que la BD apparaît beaucoup plus amusante que les livres académiques. En bref, les BD constituent de véritables vecteurs d'ouverture sur le savoir, voire une source de motivation réelle.

\section{3 À propos de l'observation et des entretiens}

L'observation et les entretiens révèlent qu'il est rare de trouver un participant qui ne possède pas un manga, un manhua ${ }^{5}$ ou qui ne lise pas une BD hebdomadaire en langue chinoise ou anglaise. Quatre occupent leurs loisirs à dévorer des mangas. La plupart de nos sujets ont tiré profit des activités autour de la BD pour enrichir leurs connaissances, améliorer leur motivation et le plaisir d'apprendre. Par ailleurs, si une BD leur plaît, ils la lisent et la relisent plusieurs fois. Les activités ont stimulé, suscité et augmenté leur curiosité. On a noté également la richesse et la précision de leur vocabulaire (surtout le vocabulaire qui apparaît un certain nombre de fois). En effet, plus on rencontre un mot, plus on le retient. Qui plus est, elle a dynamisé les interactions entre apprenants durant les séances et l'ambiance était plus relaxe.

\subsection{Quelques implications}

$\mathrm{Au}$ vu des résultats esquissés dans la présente section, bien que l'étude ne soit pas encore bouclée, on peut affirmer que l'immense majorité de nos sujets sont motivés et ont des attitudes positives envers la bande dessinée. De plus, cette dernière a joué un rôle de facilitatrice tout en leur permettant d'accéder à un niveau de compréhension plus élevé : les trois groupes expérimentaux ont obtenu de meilleurs résultats avec la $\mathrm{BD}$ complète. L'intérêt qu'ils portent à ce document authentique en tant que support, et le plaisir qu'ils prennent à apprendre avec celuici sont des atouts indéniables pour l'enseignement du FLE dans le pays. Ils peuvent affecter également l'intensité et la pérennité de leur engagement dans des situations d'apprentissage, la profondeur de leur compréhension. L'association BD avec l'enseignement du FLE pourrait donc permettre d'améliorer leurs compétences générales, linguistiques, sociolinguistiques, pragmatiques, la variété des discours, voire cultiver plaisir d'apprendre et bonheur. La BD contient des informations multimodales linguistiques et non-linguistiques qui peuvent contribuer à réduire le coût acquisitionnel de la langue cible, optimiser leur apprentissage. La variété des thèmes abordés peut favoriser le développement d'un éventail de schémas, de scripts spécifiques, auxquels adhère la communauté des locuteurs natifs de la langue française. Ces schémas et ces scripts, ces représentations de situations réelles, hypothétiques ou imaginaires leur permettront de mieux comprendre, de mieux fonctionner dans un environnement communicationnel francophone, et même d'être exposés à une considérable gamme de registres oraux et écrits. Cela leur évitera, par exemple, des malentendus culturels avec les interlocuteurs natifs. En effet, elle peut constituer pour eux, une véritable immersion dans les cultures

\footnotetext{
${ }^{5}$ Le terme chinois pour désigner la bande dessinée
} 
francophones, leur permettant d'être en contact avec la langue dans son authenticité, de mieux comprendre certains comportements divergents ainsi que le développement de leur créativité. En fait, les bandes dessinées renferment des scénarios, et reflètent le savoir pertinent à des situations de communication typique. La bande dessinée, par ses aspects distrayant, ludique, ses richesses culturelles, grammaticales et lexicales, est un bon outil pour aider les apprenants taïwanais à mieux communiquer dans la langue de Molière, à un meilleur apprentissage, à s'ouvrir sur les cultures d'expression française, à varier les formes de travail. Grâce aux caractéristiques expressives de la BD (dessins, textes, etc.), ils peuvent développer, améliorer leurs compétences lexicale, grammaticale, sémantique, orthographique, voire leurs stratégies communicatives, par exemple leur compétence de communication non-verbale (qu'on délaisse fréquemment durant le processus d'enseignement/apprentissage), tout en dotant leur discours de vivacité et de spontanéité.

La BD offre des potentialités d'exploitation pédagogique multiples et variées pouvant embrasser l'ensemble des compétences, ainsi que convenir aux diverses pratiques d'enseignement de langue étrangère. On peut l'utiliser comme un manuel de lecture en FLE. En effet, on pourrait transformer les manuels de lecture existants, les ouvrages qui proposent des activités linguistiques en bandes dessinée ou en roman graphique ${ }^{6}$, c'est-à-dire mettre à la disposition des apprenants, surtout de niveau A1 et A2, des manuels en bandes dessinées. Ces manuels d'apprentissage au format BD seront élaborés en étroite collaboration entre les enseignants sinophones et francophones. Cela les inciterait à la lecture, faciliterait leur compréhension, surtout la compréhension des scripts plus diffus, comme parler de sport, de la politique, d'émotions, puis améliorera leurs compétences de lecture. De par son style conversationnel, elle constitue, par exemple, un moyen très attrayant pour enseigner ou prendre contact avec les onomatopées, pour enseigner explicitement la variété des structures rhétoriques. C'est également un bon matériel pour travailler sur les expressions familières, les principaux registres de langue, les nuances que transmettent certains mots, l'humour des Français et des Francophones, et même approcher un sujet complexe sans ennuyer. En d'autres termes, elle les prépare à une communication authentique et efficace. D'ailleurs, plusieurs éditeurs tels que Clé international, Bordas, Press Pocket Books, Harrap's, Nathan, etc., l'ont bien compris en se servant déjà de la $\mathrm{BD}$ pour les langues étrangères. De même, certaines entreprises et institutions reconnaissent ses vertus pédagogiques en lui faisant appel de temps en temps pour résoudre des problèmes de communication.

Ce média complexe peut servir d'appui à l'imagination, la reconstitution d'histoire à partir des vignettes. On pourrait demander aux apprenants de recomposer la séquence d'une histoire, d'imaginer la suite de l'épisode, de résumer le contenu, soit oralement, soit par écrit, etc. Cela développera leurs capacités d'observation, d'interprétation, de même que leur esprit critique. En effet, sur la même page, se côtoient différents types d'écriture : les bulles, les narrations et les dessins.

De la même manière, la bande dessinée, étant un instrument puissant de transmission de la culture, pourrait aider les apprenants à se familiariser avec certains aspects des cultures francophones, favoriser l'apprentissage des relations interculturelles. En fait, la lecture d'une BD francophone n'est autre chose qu'un contact avec les cultures d'expression française. On pourrait, à partir d'une BD francophone, leur faire comprendre certaines références culturelles françaises ou francophones qui leur sont incompréhensibles, discuter un fait social différent ou analyser certains clichés qui en découlent. Ainsi, grâce à ces nouveaux écrits, les apprenants peuvent accéder à de nouveaux savoirs non envisagés dans le cursus, avancer à leur rythme. Lire une bande dessinée, c'est découvrir à chaque instant quelque chose de nouveau. Une bonne bande dessinée vaut parfois mieux qu'un long discours de l'enseignant.

\footnotetext{
${ }^{6}$ C'est un genre littéraire entre le roman et la BD.
} 
Cet outil de communication efficace peut être une source de motivation, un moyen amusant pour motiver, pour apprendre dans la bonne humeur. On peut s'en servir pour faire de la maïeutique, c'est-à-dire faire accoucher leur connaissance, déclencher l'expression, etc. Pour susciter un débat en classe, on peut recourir aux vignettes de certaines revues francophones. De même, on peut expliquer des mots, des expressions à partir de la BD. On peut même inventer des jeux, comme les jeux d'observation (par exemple le jeu des sept erreurs de Djamel, 2007 : 238-239). Bref, elle peut être le point de départ d'activités orales ou écrites dans la classe de langue.

Ce type de moyen d'expression universelle peut être une aide à la traduction, à l'autonomie. On peut recourir à cet outil pédagogique pour faire voir aux apprenants, les différences importantes entre le chinois mandarin et la langue cible, autrement dit, effectuer une analyse contrastive de la traduction du français vers le chinois et vice versa. D'ailleurs, comme souvent les bandes dessinées (par exemple Tintin, Astérix, entre autres) sont disponibles dans leur L1, ils peuvent s'exercer ou travailler à la maison avec l'aide des deux versions. L'apprenant y trouvera la satisfaction de lire seul, de traduire sans l'aide de l'enseignant, acquérant ainsi son autonomie de lecteur, de traducteur (tout en respectant son rythme individuel).

On peut utiliser ce document authentique pour déclencher la créativité, la confiance en soi. Les apprenants peuvent jouer, mettre en scènes les dialogues de la $\mathrm{BD}$, trouver un titre, imaginer le contenu des bulles, reconstituer le " non dit ", s'inventer des histoires sur les situations suggérées par les dessins, etc. C'est à juste titre qu'on l'a qualifié de " langage libérateur " (Baron-Carvais 1994 : 78-79). Il peut permettre de briser la monotonie, le manque de réalisme des manuels académiques, voire développer leur esprit critique. Il peut les accompagner dans et hors l'école, voire rester disponible longtemps. Grâce à la BD, ils peuvent rester en contact avec la LE, de même qu'entretenir régulièrement leur niveau de langue.

\section{$5 \quad$ Pour conclure provisoirement}

L'objectif principal de cet article était d'évaluer les attitudes des apprenants taïwanais de FLE à l'égard de la bande dessinée. Pour ce faire, on a eu recours à un questionnaire, des entretiens, mais aussi à des tâches à partir des bandes dessinées. Les résultats de notre contribution montrent que l'immense majorité de nos sujets ont des attitudes positives à l'égard de la bande dessinée, cet outil d'aide à l'apprentissage, et qu'ils ont saisi mieux les activités à partir de cet outil pédagogique. Quoi qu'il en soit, le bilan final se révèle satisfaisant, tant au plan des compétences linguistiques, culturelles qu'interculturelles. De plus, il indique que l'attitude n'est pas seulement une variable unidimensionnelle, mais aussi multidimensionnelle. Par la même occasion nous en avons profité pour esquisser quelques pistes et propositions.

Somme toute, il serait aberrant de passer sous silence ce penchant des apprenants pour le neuvième art. Dans les conditions de l'expérimentation, la bande dessinée apparaît, de façon notoire, comme un outil qui peut accompagner l'apprentissage du FLE à Taiwan, un facteur de facilitation de l'apprentissage. Elle peut donc se tailler une niche dans le kaléidoscope des matériels pédagogiques et didactiques. Toutefois, son utilisation doit être complémentaire du discours de l'enseignant, des autres supports les plus en vogue comme les TIC, les réseaux sociaux, le cinéma ; sans oublier la réalité du travail individuel que l'apprenant doit faire pour s'approprier la LC.

La présente contribution, de caractère exploratoire, ne prétend pas épuiser l'ensemble des questions soulevées. Nous avons seulement voulu souligner l'importance que revêt l'utilisation de la bande dessinée comme outil pédagogique dans le contexte taïwanais. Mais il est possible que certaines réalités aient échappé à l'analyse. En d'autres termes, il sera judicieux à l'avenir, par exemple, de travailler avec un échantillon plus représentatif, d'élargir la recherche aux 
apprenants taïwanais d'espagnol et d'allemand, d'analyser les différences d'attitudes entre les sexes, voire tenir compte de la classe socioprofessionnelle des parents.

\section{Références}

Ajzen, Icek/Fishbein, Martin (1977): "Attitude-behavior relations: A theoretical analysis and review of empirical research". Psychological Bulletin 84/5: 888-918.

Ajzen, Icek/Fishbein, Martin (1980): Understanding attitudes and predicting social behavior. New Jersey.

Armitage, Christopher J. (2003): "Beyond attitudinal ambivalence: effect of belief homogeneity on attitude-intention-behavior relations". European Journal of Experimental Social Psychology 33: 551-563.

Barbieri Durao, Adja Balbino de Amorin (2004): "¡Los tebeos en pantalla!: el empleo del "tebeo" en el proceso de enseñanza/aprendizaje de español como lengua extranjera". In: Perdiguero Villarreal, Hermógenes/Álvarez, Antonio A. (coord.): Acta del X Congreso Internacional de ASELE. Burgos: 596-613.

Barron-Carvais, Annie (1994): La bande dessinée. Paris.

Cacioppo, John T./Petty, Richard E. (1982): "The need for cognition". Journal of Personality and Social Psychology 42: 116-131.

Campbell, Bob (2001): "Pupils' perceptions of science education at primary and secondary school". In: Behrendt, H. et al. (éds.): Research in Science Education - Past, Present and Future. Dordrecht: $125-130$.

Clark, Richard E. (1994): "Media will never influence learning". Educational Technology, Research and Development 42/2: 21-29.

Clément, Richard et al. (1994): "Motivation, self-confidence and group cohesion in the foreign language". Language Learning 44/3: 417-448.

Cloutier, Richard (2003): "La réussite scolaire des garçons: un défi à multiples facettes". Vie Pédagogique 127: 9-12.

Conner, Mark/Sparks, Paul (2002): "Ambivalence and attitudes". European Review of Social Psychology 12: 37-70.

Cutrim Schmid, Euline/Schimmack, Estelle (2010): "First steps towards a model of interactive whiteboard training for language teachers". In: Thomas, Michael/Cutrim Schmid, Euline (éds.): Interactive Whiteboards: Theory, Research and Practice. Hershey/ PA.

Dewaele, Jean-Marc/Regan, Vera (2002): "Maîtriser la norme sociolinguistique en interlangue française: le cas de l'omission variable de "ne"". Journal of French Language Studies 12/2: 123-148.

Djamel, Bendiha (2007): "La bande dessinée comme support didactique dans l'enseignement du FLE". Synergies Algérie 1: 235-240.

Dörnyei, Zoltán (1994). "Motivation and motivating in the foreign language classroom". Modern Language Journal 78/3: 273-284.

Eagly, Alice H./Chaiken, Shelly (1993): The psychology of attitudes. Orlando/FL.

Ellis, Rod (1992): "Learning to communicate in the classroom: A study of two language learners' request." Studies in Second Language Acquisition 14/1: 1-23.

Executive Yuan (2003): Challenge 2008: National development plan (2002-2007). Taipei.

Fazio, Russell H. (1986): "How do attitudes guide behavior?". In: Sorrentino, Richard H./Higgins, Edward Tory (éds.): The handbook of motivation and cognition: Foundation of social behavior. New York: 204-243.

Fazio, Russell H. (1989): "On the power and functionality of attitudes: The role of attitude accessibility". In: Pratkanis, Antony R. et al. (éds.): Attitude structure and function. Hillsdale/NJ: $153-179$.

Fishbein, Martin/Ajzen, Icek (1975): Belief, attitude, intention and behavior: An introduction to theory and research. New York. 
Galvis, Coromoto (2010): "Actitud hacia el bilingüismo inglés-español en estudiantes de secundaria norteamericanos". Revista Nebrija de Lingüística Aplicada 8/4: 96-99.

Gardner, Robert C. (1985): Social psychology and second language learning: The role of attitudes and motivation. Londres.

Gass, Susan M. et al. (1998): "The role of input and interaction in second language acquisition. Introduction to the special issue". Modern Language Journal 82/3: 299-307.

Havard, Neil (1996): "Student attitudes to studying A-Level sciences". Public Understanding of Science 5/4: 321-330.

Huang, Shing-Ying (2005): "Taiwanese students talk about English in Taiwan and their lives". Languages, Communities, and Education Spring 2005: 45-54.

Jonas, Klauss et al. (1997): "Effects of attitudinal ambivalence on information processing and attitude-intention consistency". Journal of Experimental Social Psychology 33/2: 190-210.

Kasanga, Luanga A. (1996): "Peer interaction and second language learning". Canadian Modern Language Review 52/4: 611-639.

Larsen-Freeman, Diane/Long, Michael H. (1994): Introducción al estudio de la adquisición de lenguas. Madrid.

Leather, Sue (2003): "Taboos and issues". ELT Journal 57/2: 205-206.

Legendre, Renald (1993): Dictionnaire actuel de l'éducation. Montréal.

Lescure, Richard et al. (2006): DELF A2 200 activités. Paris.

Lewis, Gordon (2010): Bringing technology in the classroom. Oxford.

Lin, Mei-Chih (2007): El aprendizaje y el uso del pretérito imperfecto y del pretérito indefinido de indicativo por estudiantes taiwaneses de español como lengua extranjera. Thèse de doctorat. Universidad de Alcalá.

Luis Banegas, Darío (2011): "Comment: Teaching more than English in secondary education". ELT Journal 65/1: 80-82.

Marcos-Llinas, Mónica (2007): "Variables afectivas en la clase de lenguas extranjeras". Interlinguística 17: 676-678.

Marton, Philippe (éd.) (1999): "Les technologies de l'information et de la communication et leur avenir en éducation". Education et francophonie 27/2. Disponible sur: http://www .acelf.ca/c/revue/pdf/EF-27-2-WEB.pdf [consulté le 10 août 2010].

Morlat, Jean-Marcel/Tomimoto, Janina (2007): "La bande dessinée en classe de FLE". ÉduFLE.net. Disponible sur: www.edufle.net/La-bande-dessinee-en-classe-de-FLE [consulté le 28 juillet 2010].

Osborne, Jonathan/Collins, Sue (2000): Pupils' and parents' views of the school science curriculum. Londres.

Petrof, John V. (1999): Comportement du consommateur et marketing. Québec.

Postel-Vinay, Olivier (2002): "Les jeunes et la science : les filles se distinguent". La Recherche 359: 46-51.

Potvin, Pierre/Paradis, Louise (2000): Facteurs de réussite dès le début de l'éducation préscolaire et primaire. Québec.

Rogers, Donna L. (2000): "A paradigm shift: Technology integration for higher education in the new millennium". Educational Technology Review 13: 19-27.

Romero Trillo, Jesús (2002): "The pragmatic fossilization of discourse markers in non-native speakers of English". Journal of Pragmatics 34/6: 769-784.

Russell, Thomas L. (1999): The no significant difference phenomenon. North Carolina.

Saillard, Claire (1998): "Quand variété veut rimer avec officialité, ou la situation sociolinguistique de Taiwan". Revue Française de Linguistique Appliquée 3/1: 87-99.

Salins, Geneviève-Dominique de/Dupré La Tour, Sabine (1988): Exercices de grammaire perfectionnement. Paris.

Sjoberg, Svein (2000): "Science and scientist: The SAS-study cross cultural evidence and perspectives on pupils' interests experiences and perceptions-background: development 
and selected results". Acta didactica 1. Oslo. Disponible sur: http://folk.uio.no/sveinsj [consulté le 21 octobre 2010].

Slate, John R. et al. (2002): "The "digital" divide: Hispanic college students' views of educational uses of the Internet". Assessment and Evaluation in Higher Education 27/1: 75-93.

Sossouvi, Laurent-Fidèle (2006): "Analyse des facteurs affectifs intervenants dans l'apprentissage de français langue étrangère: le cas des apprenants taïwanais". $l^{\text {er }}$ Congrès Régional Asie-Pacifique CAP - FIPF. Taipei.

Sossouvi, Laurent-Fidèle (2010): "L'apprenant sinophone entre plusieurs langues européennes : à la recherche d'une langue passerelle". Journal of European Languages 3: 22-44.

Sparks, Paul et al. (2001): "Ambivalence about health-related behaviors: an exploration in the domain of food choice". British Journal of Health Psychology 6: 53-68.

Tarone, Elaine (2000): "Still wrestling with 'context' in interlanguage theory." Annual Review of Applied Linguistics 20: 182-198.

Thomas, Raymond/Alaphilippe, Daniel (1993): Les attitudes. Paris.

Tragant, Elsa/Muñoz, Carmen (2000): "La motivación y su relación con la edad en un contexto escolar de aprendizaje de una lengua extranjera". In: Muñoz, C. (éd.): Segundas lenguas: adquisición en el aula. Barcelona: 81-105.

Usun, Salih (2003): "Educational uses of the Internet in the World and Turkey: A comparative review". Turkish Online Journal of Distance Education 4/3. Disponible sur: http://tojde.anadolu.edu.tr/tojde11/articles/usun.htm [consulté le 28 juillet 2010].

Venturini, Patrice (2004): "Attitudes des élèves envers les sciences : le point des recherches". Revue française de pédagogie 149: 97-123.

Wisher, Robert A./Priest, Annette N. (1998): "Cost-effectiveness of audio teletraining for the US Army National Guard". The American Journal of Distance Education 12/1: 38-51.

Yager, Kent (1998): "Learning Spanish in Mexico: The effect of informal contact on student attitudes of language gain". Hispania 81/4: 889-911.

Yang, Shu Ching/Chen, Yi-Ju (2007): "Technology-enhanced language learning: A case study". Computers Human Behavior 23/1: 860-879. 
Annexe

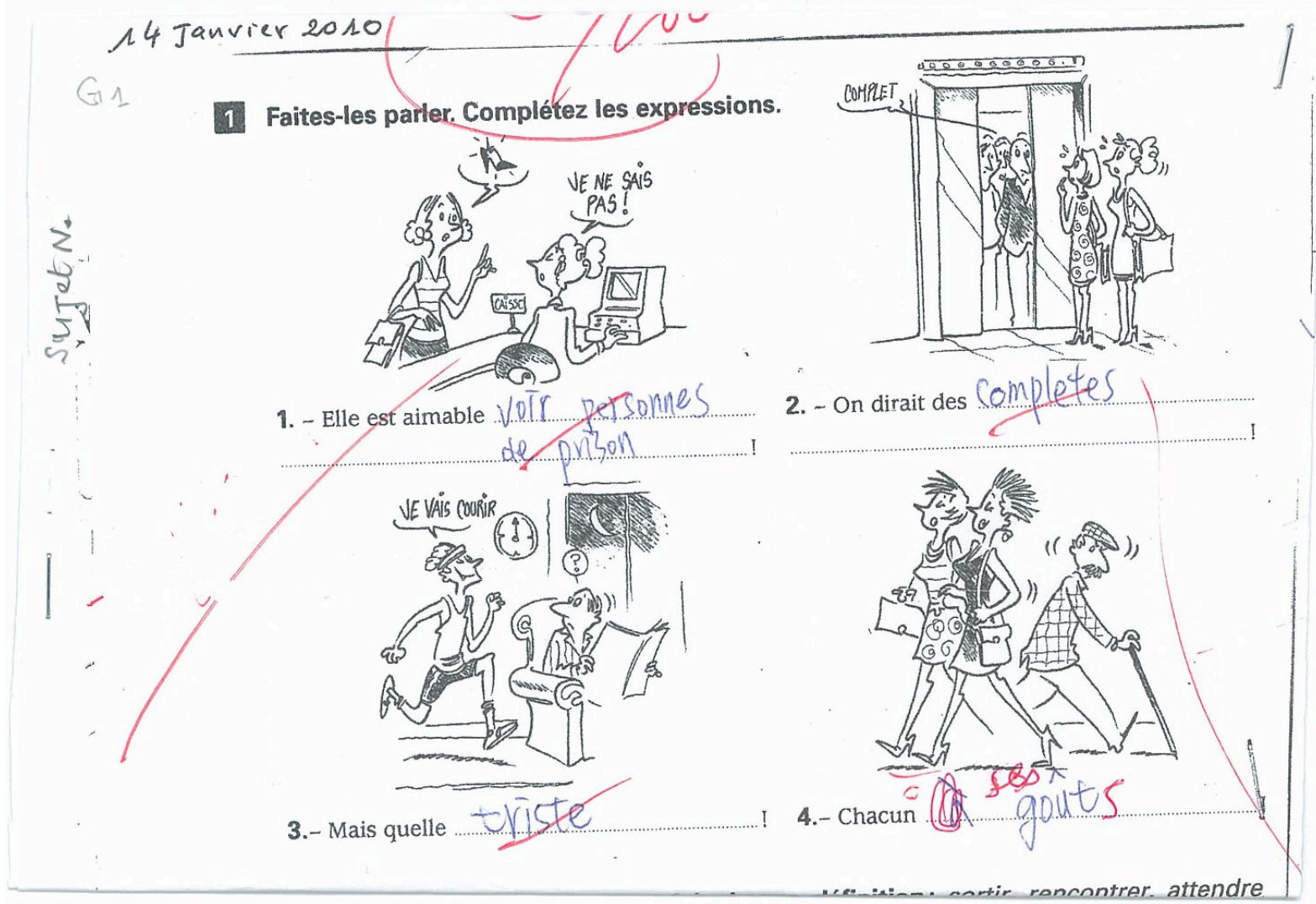

\section{Nom: Ivumero:}

Faites-les parler.

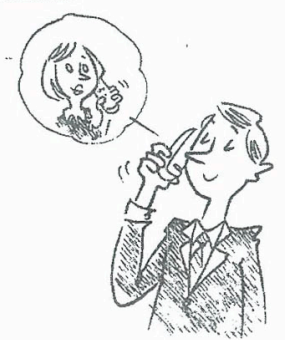

1. - Allô? Monsieur Suter à l'appareil. Je voudrais parler à monsieur Mougel.

- Ne guittez pas s'il vou plaqt.

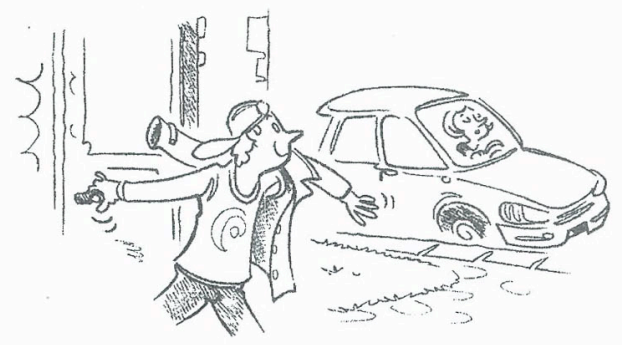

3. - Dépêches- toi!

J'arrive! J'arrive!

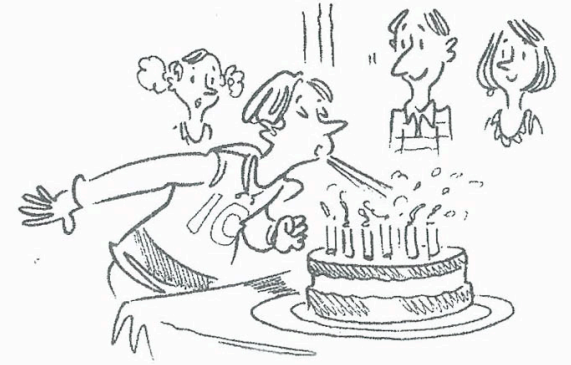

2. - Bon antivesere

- Oh! Merci!

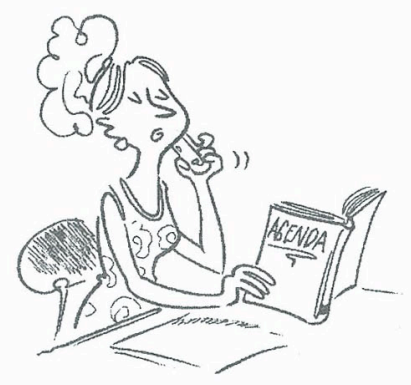

4. - Tu fais quoi demain soir?

- Je ne sais pas, peut - suis _........ sur mon agenda. Super, je suis libre. 


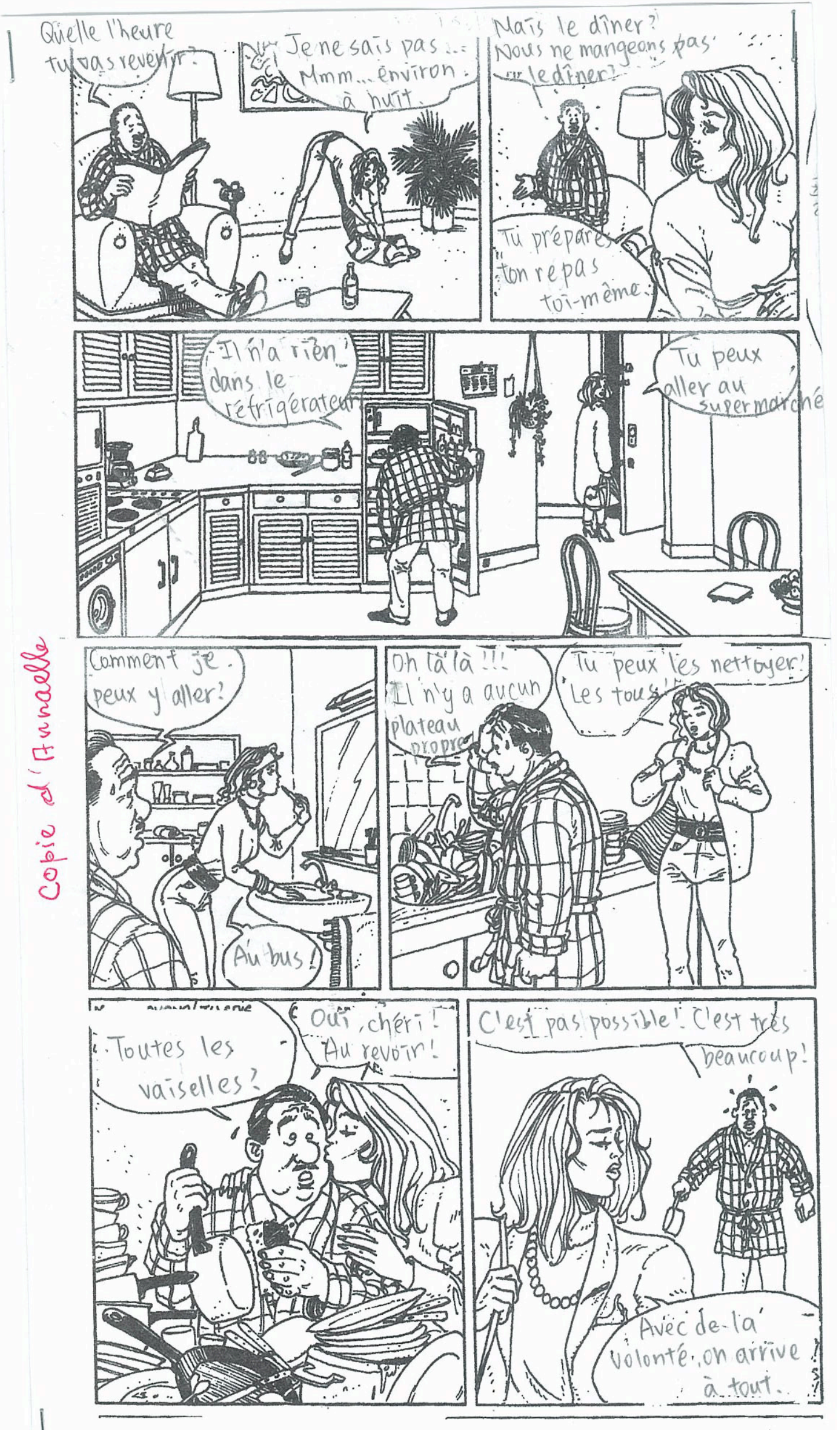



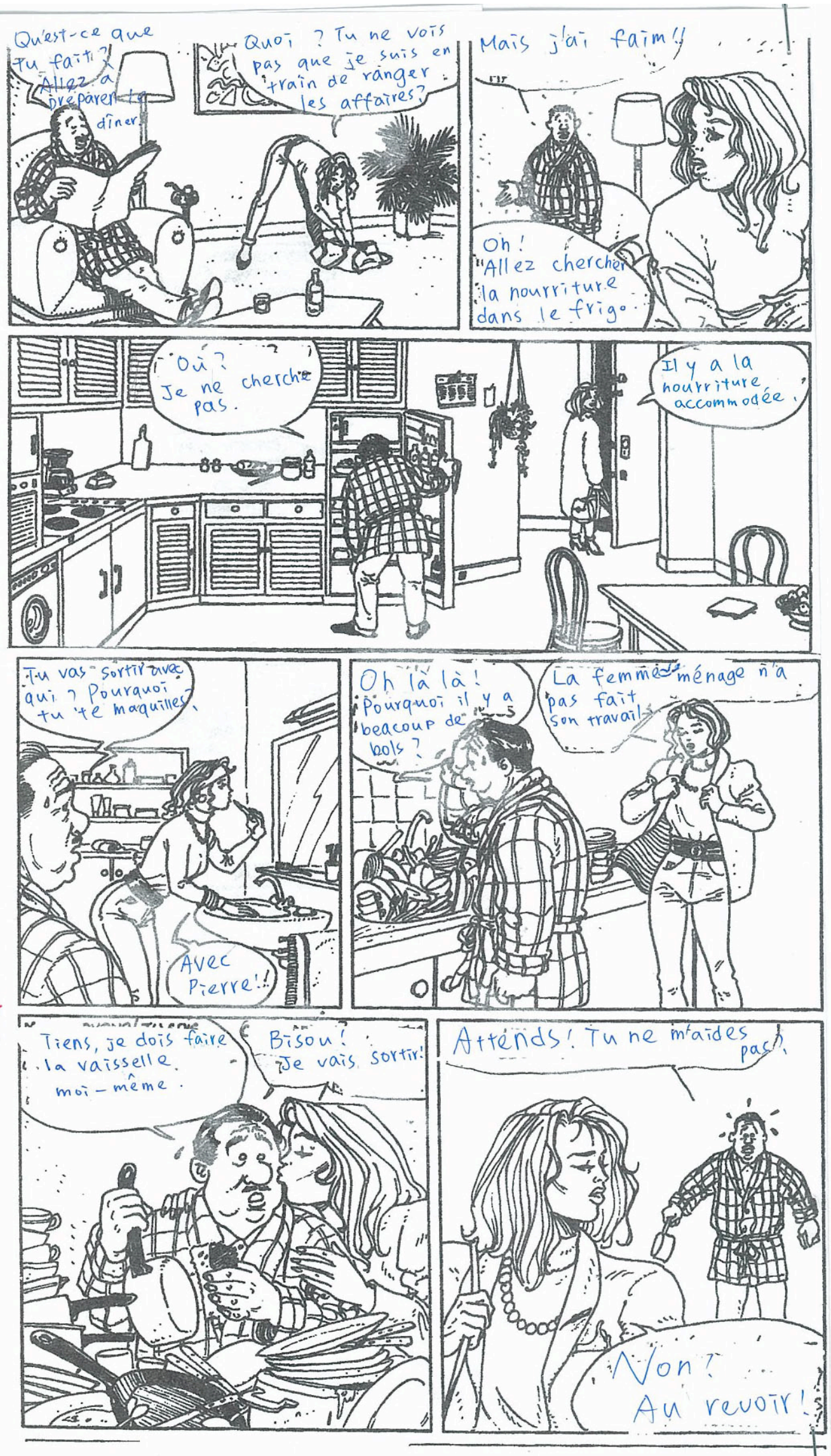

Annexe 2: Deux copies des participants du groupe G2 avec la tâche:

" À partir de la planche de BD suivante, imaginez les paroles de chaque vignette. " 

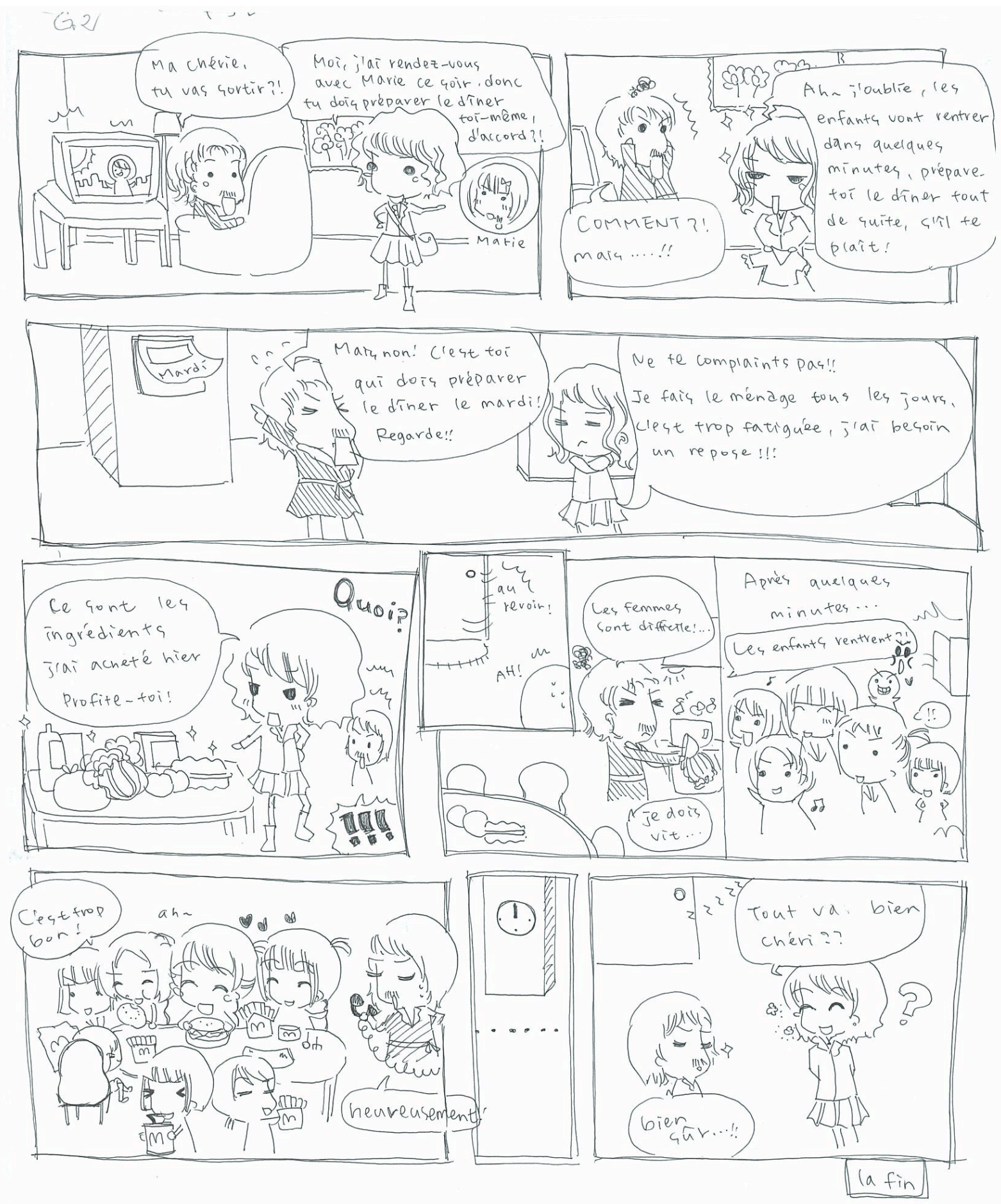

Annexe 3: Une planche de BD réalisé par l'une de nos participantes, Peggy, sur le thème:

" La vie d'une famille nombreuse " 


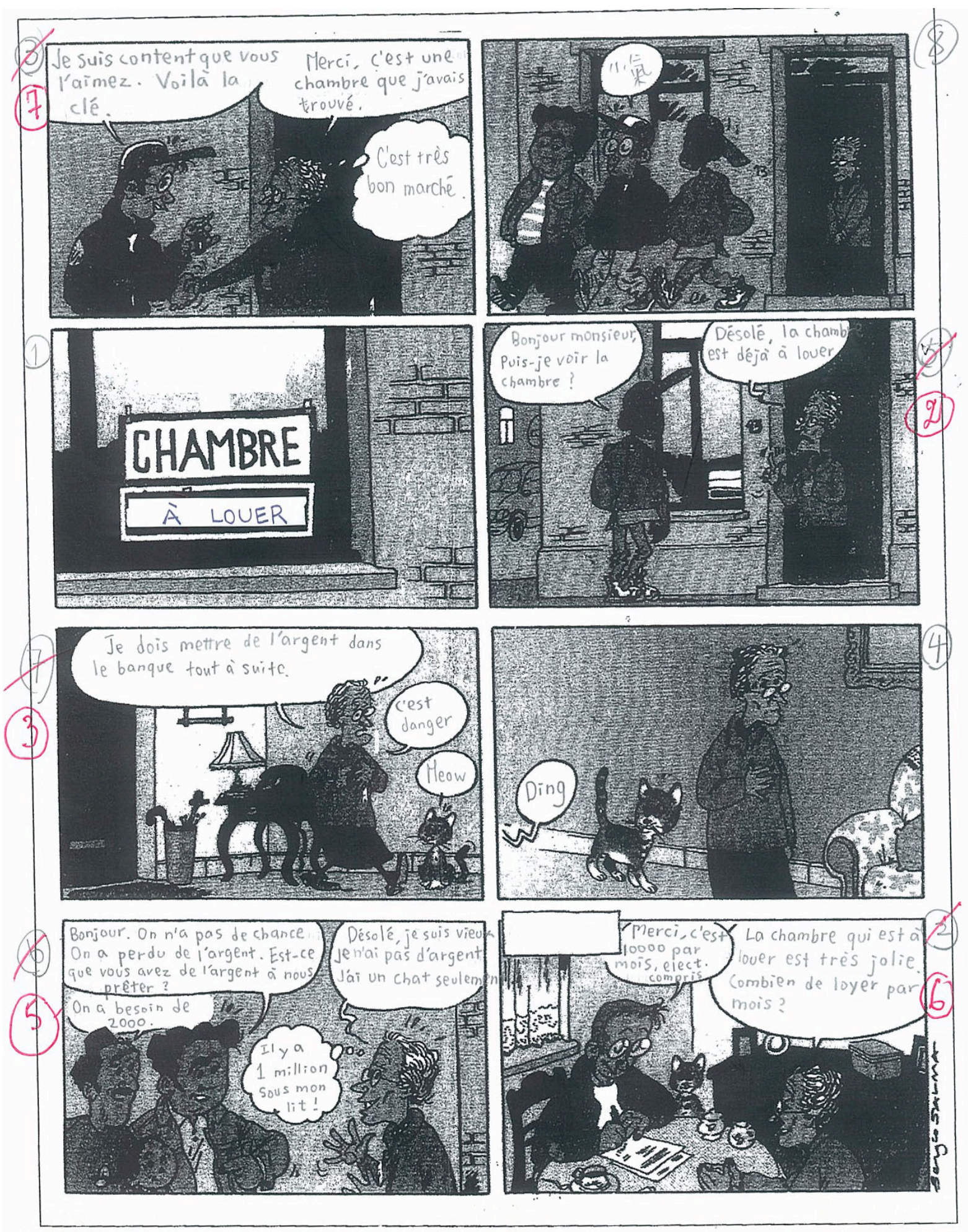

Annexe 4: Une copie des participants du groupe G3 avec la tâche: " À partir de la planche de BD suivante intitulée ' Préjugés ', retrouvez l'histoire en remettant les vignettes dans l'ordre. Imaginez les paroles de chaque vignette." 


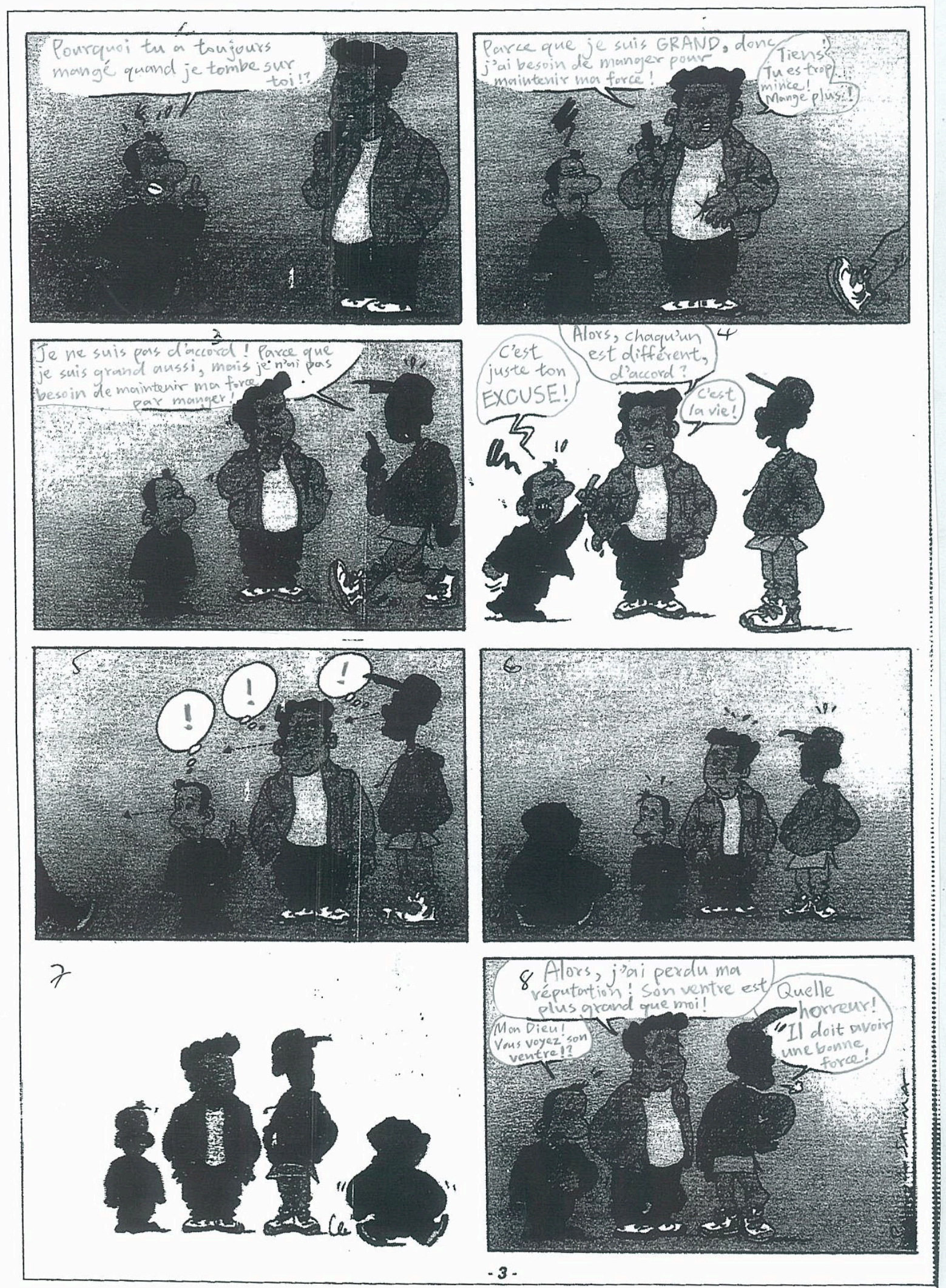

Annexe 5: Une copie des participants du groupe G3 avec la tâche: " À partir de la planche de BD suivante intitulée ' Un monde de différences ', imaginez des paroles de chaque vignette. Pour vous aider, faites la description des personnages de cette BD... Imaginez la situation. " 